\title{
Invisible Higgs in weak bosons associative production with heavy quarks at LHC: probing mass and width
}

\author{
E. E. Boos*, \\ Skobeltsyn Institute of Nuclear Physics, Moscow State University, \\ Vorobiovy gory, Moscow 119991, Russia \\ S. V. Demidov'and D. S. Gorbunov ${ }^{\ddagger}$ \\ Institute for Nuclear Research of the Russian Academy of Sciences, \\ 60th October Anniversary prospect 7a, Moscow 117312, Russia
}

\begin{abstract}
New physics coupled to the Higgs boson may hide it in the standard decay channels to be investigated at LHC. We consider the models where new invisible dominant decay modes of the Higgs boson are responsible for this hiding. We propose to study at LHC the weak boson production associated with heavy quarks: our analysis revealed that boson pair invariant mass distribution is sensitive to both mass and width of the invisible Higgs boson, if it is not too far from the weak boson pair threshold. We present tree-level results for the most relevant cases of top quarks and of bottom quarks in Standard Model extensions with large $b$-quark Yukawa coupling. We argue that QCD corrections do not spoil these results allowing for unambiguous extraction of the Higgs boson mass and width from the analysis of large enough amount of data.
\end{abstract}

\footnotetext{
*e-mail: boos@theory.sinp.msu.ru

$\dagger$ e-mail: demidov@ms2.inr.ac.ru

‡e-mail: gorby@ms2.inr.ac.ru
} 


\section{Introduction}

The main task to accomplish at LHC is searching for the Higgs boson, the only particle of the Standard Model (SM) has not been observed so far. However, it has become clear that the SM itself is incomplete. There are neutrino oscillations, strong CP-problem, dark matter, baryon asymmetry of the Universe which lack for explanation within the SM (see, e.g. review section of Ref. [1]).

In the SM only the Higgs sector remains still hidden, while all others were thoroughly explored without any convincing direct evidence of new physics processes in there besides neutrino oscillations. So, it can happen that at LHC a new physics will show up (for the first time?) right in the Higgs sector. Generally, in SM extensions both the Higgs boson production and decay rates deviate significantly from the SM prediction. May be the simplest yet physically-motivated extension of this type is the model of dark matter scalar particles [2]. This extension contains one new scalar field singlet under the SM gauge group. Parity ensures that the new scalar particle is stable and hence the dark matter candidate. The only renormalizable interaction of this scalar with SM particles is coupling to the SM Higgs field ${ }^{1}$. This coupling is responsible for the dark matter generation in the early Universe due to Higgs boson scatterings in primordial plasma. Thus, the (virtual) SM Higgs boson can decay into two dark matter particles. Appearance of this invisible (real or virtual) decay mode is the only tree-level modification to be searched for at LHC in this simple model.

There are many other physically-motivated and (much) more complicated extensions of the SM where properties of the Higgs boson get modified [4]. Among them are models with additional spatial dimensions suggesting a solution to the problem of hierarchy between the electroweak and Plank scales $[5,6]$. There are also multi-Higgs models capable of explaining the baryon asymmetry of the Universe, see e.g. [10]. Supersymmetric extensions of the SM address both problems and also have (natural) candidate(s) to be dark matter particles. In all the models above, as well as in many other extensions of the SM, the Higgs boson starts to interact with new fields. This changes its production rates and decay pattern to an extent which in some

\footnotetext{
${ }^{1}$ In this type of models the new scalar can also couple to other new (otherwise hidden) fields, e.g. can transform non-trivially under some new (hidden) gauge group. In literature models of this kind are dubbed as Higgs portal [3], as the only door to the hidden fields is in the Higgs sector.
} 
cases (see, e.g. $[4,7,8,9])$ becomes crucial for the prospects of a particular experiment in searches for the Higgs boson.

Let us consider a class of such extensions of the SM where new invisible Higgs decay mode dominates. In these models the branching ratios of all visible decay modes of the Higgs boson get decreased, sometimes to the level beyond the LHC sensitivity (see, e.g., discussion in Refs. [11, 12]). The question is how to search for the Higgs boson and measure its major parameters mass and width in this case, where the Higgs boson is invisible itself.

The strategy for hunting the invisible Higgs boson at LHC has been developed in literature to a certain extent. In particular, the Higgs boson production with subsequent decay to invisible mode gives rise to missing $P_{T}$ signature in various channels such as Vector Boson Fusion $q q \rightarrow q q H$ [13] and the associated production processes, $g g \rightarrow t \bar{t} H$ [14] and $q q \rightarrow Z H$ or $q q \rightarrow W^{ \pm} H[15,16]$. A tricky question here is how to make sure that the observed signal is really due to production of the Higgs boson, not some other particle. Another disadvantage of the missing $P_{T}$ signature is that only the Higgs boson mass can be estimated from the data analysis: the Higgs boson width remains unobservable.

To address both issues one can consider the very channels where the exchange of the Higgs boson restores unitarity in SM particle collisions. These are channels with massive vector bosons in initial and/or final states $[17,19,18]$. The relevant processes at LHC are inclusive $Z Z$ and $W^{+} W^{-}$ production. In case of the Higgs boson mass above the weak boson pair thresholds these processes have been thoroughly studied [20]. The Higgs boson mass can be reconstructed, e.g., from analysis of invariant mass of outgoing final leptons, while measurement of the inclusive production rate yields the decay branching ratio to weak bosons. The latter implies the estimate of the total Higgs boson width (saturated by invisible mode) assuming the SM coupling between the Higgs and weak gauge bosons.

In this paper we are interested mostly in the opposite case where the Higgs boson decays to weak bosons are kinematically forbidden on shell. This case of the light Higgs boson is certainly favorable from the combined analysis of electroweak precision measurements $[1,27]$. We concentrate on the models, where the Higgs boson width is dominated by the invisible mode, hence the Higgs branching ratios into SM particles are suppressed with respect to the estimates within the SM. In this setup virtual Higgs boson contributions to $W^{+} W^{-}$and $Z Z$ production via weak boson fusion and gluon fusion have been already considered in literature, see, e.g., [21]. 
The main observation of this work is that the measurements of total cross section and invariant mass distribution of the weak boson pair generally allow to estimate both the Higgs boson mass and width. This statement is illustrated by the realistic example of the Higgs boson contribution to the weak boson production associated with $t$-quarks: $p p \rightarrow Z Z \bar{t} t, p p \rightarrow W W \bar{t} t$. Likewise we study processes $p p \rightarrow Z Z \bar{b} b$ and $p p \rightarrow W W \bar{b} b$. The production rates are too small here, but might be of some interest in the SM extensions where the Higgs boson coupling to $b$-quark is amplified, e.g., in multi-Higgs or supersymmetric models. The dependence of the total cross section and the gauge boson invariant mass on the Higgs boson parameters is explained analytically in this example of associated production with $b \bar{b}$-pair.

As the first step towards this goal we discuss in this paper only signal properties, leaving the so called reducible background without any discussion. Thus, we consider tree level contribution of Higgs boson and all other SM particles to the processes presented above: production of two weak bosons and two heavy quarks in proton-proton scattering. Meanwhile, we estimated the size of possible uncertainties in the obtained results due to QCD quantum corrections and found them small. Finally, we expect that the invariant mass of weak boson pairs in other relevant channels, like $Z Z$ and $W^{+} W^{-}$or $j j Z Z$ and $j j W^{+} W^{-}$is also sensitive to both the SM Higgs boson mass and width, but leave the corresponding study for future work. Note that the dependence of the invariant mass on the Higgs mass in these channels has been considered in literature (see, e.g., [21]).

As a side remark, come back to the fundamental role of the Higgs boson in particle scatterings, which exchange restores unitarity in the electroweak theory. To the weak boson production associated with heavy quarks we are considering here, both gauge and Yukawa Higgs couplings contribute. Hence, its study allows to test the Higgs mechanism both in the gauge and in the fermion sectors. This is an important task and it is worth to be performed at LHC and SLHC even if the Higgs boson signal would closely resemble what SM predicts.

The paper is organized as follows. In Section 2 we discuss the weak boson pair production associated with top quarks - the process which study at LHC gives a chance to measure both the width and mass of the invisible Higgs boson. We consider in Section 3 similar processes with $b$-quarks in models where $b$-quark Yukawa coupling is amplified with respect to that of in the SM. Simple analytical formulas presented in Section 4 for weak boson production in partonic scatterings of quarks illustrate the obtained results 
and provide with understanding of the physics behind. Section 5 contains summary.

\section{$2 \quad$ Invisible Higgs in $p p \rightarrow t \bar{t} Z Z$ and $p p \rightarrow t \bar{t} W^{+} W^{-}$ at $\mathrm{LHC}$}

We begin our study with the channel $p p \rightarrow t \bar{t} Z Z$. We would like to consider virtual contribution of the Higgs boson to these processes, since it remains almost the same as in the SM. At the same time, as it is mentioned in Introduction, a large rate of invisible Higgs decay would prevent searches for the Higgs boson by means of usual methods, that is by studying Higgs boson decay modes. In these final states the Higgs contribution will be considerably suppressed by the ratio of the Higgs boson width to that within the SM.

We use CompHEP $[22,23,24]$ to calculate the tree level partonic cross sections of the process $p p \rightarrow t \bar{t} Z Z$. This process is dominated mostly by the subprocess $g g \rightarrow t \bar{t} Z Z$, where the virtual Higgs boson decays into $Z Z$. We note, that both signal (Higgs boson contribution) and SM irreducible background amplitudes are taken into account in our analysis. We adopt CTEQ6L1 [25] for parton distribution function in our calculations. The width of Higgs boson is calculated by HDECAY program [26]. No phase space cuts

are imposed on the final state. In this paper we outline general behavior of the cross section and $Z Z$ invariant mass distribution and postpone detailed calculations, including analysis of the reducible background and the detector response, for future work. It is worth to note in this respect that dealing with a final state of several heavy particles one can hope to sufficiently suppress the reducible backgrounds playing with cuts on the relevant physical observables.

The results for total cross sections and invariant mass $Z$-boson pair distributions are presented in Figures 1-4. Here and further we present results for collision energies of $\sqrt{s}=14 \mathrm{TeV}$ and $\sqrt{s}=10 \mathrm{TeV}$, for comparison. In Figure 1 we plot the dependence of the total cross section of $p p \rightarrow t \bar{t} Z Z$ channel at $\sqrt{s}=14 \mathrm{TeV}$ and $\sqrt{s}=10 \mathrm{TeV}$ on the Higgs boson mass $m_{H}$ for a set of values of the Higgs boson width $\Gamma_{H}$. Here and further we perform study of the low mass range of the Higgs boson mass preferred by the combined fit to electroweak precision measurements [1, 27]. As one expects, at large values of the Higgs boson width the virtual Higgs boson contribution to the amplitude of this process decreases and hence the total cross section also 

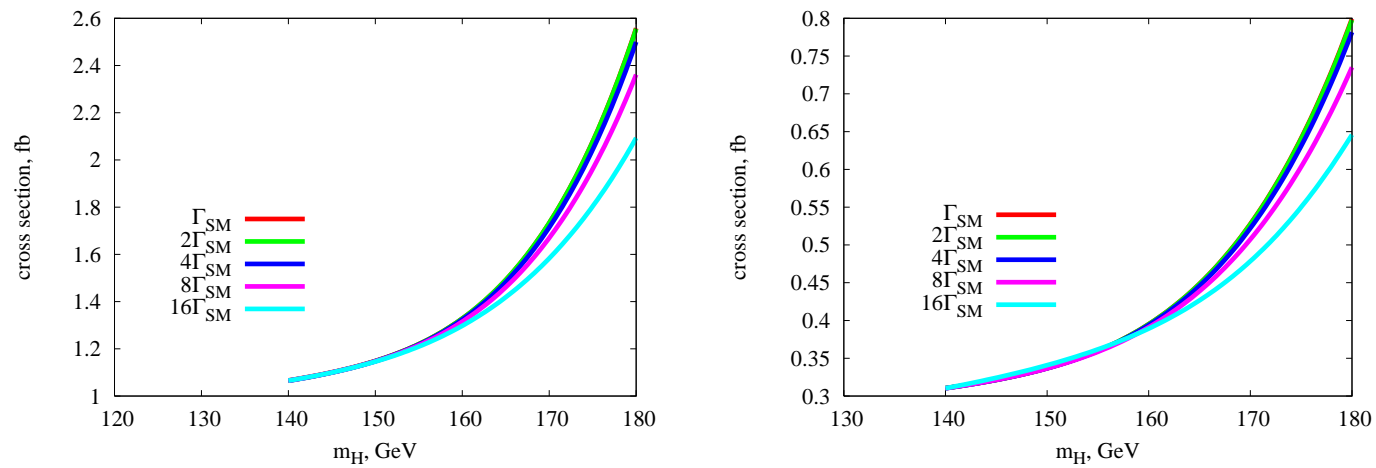

Figure 1: The dependence of the total cross section $p p \rightarrow t \bar{t} Z Z$ at $\sqrt{s}=$ $14 \mathrm{TeV}$ (left panel) and $\sqrt{s}=10 \mathrm{TeV}$ (right panel) on the mass of the Higgs boson in for a set of values of the Higgs boson width. Here $\Gamma_{S M}$ is the width of the Standard Model Higgs boson.

decreases. Figure 2 shows the corresponding invariant mass $m_{Z Z}$ distribution for different values of Higgs boson mass in the cases of the SM Higgs boson width (upper panel) and eight times larger width (lower panel). We see that both shape and position of maximum of $m_{Z Z}$ distribution strongly depend on mass $m_{H}$, which can be used to pin down the Higgs boson mass. Moreover, from Figure 3 one concludes that this $m_{Z Z}$ distribution does not depend on the width of the Higgs boson, except for the case of near threshold values of its mass (see lower panel). Even in the latter case, for $m_{H}$ near $180 \mathrm{GeV}$, we observe that the position of maximum in $m_{Z Z}$ distribution varies quite moderately with reasonable increase of the Higgs boson width.

Parameters of the Higgs boson - mass and width — can be obtained, as usual, from the combined two-parametric fit to the observables of this channels. From the analysis above, however, we suggest the following simple strategy for studying the invisible Higgs boson. One measures $m_{Z Z}$ distribution and finds the mass of the Higgs boson from the position of maximum in Figure 2. Then by using either the dependence of the total cross section on the Higgs boson width (see Figure 4) or (for the case of near threshold masses of the Higgs boson) the shape of $m_{Z Z}$ distribution we determine $\Gamma_{H}$.

Similarly one can investigate another channel, $p p \rightarrow t \bar{t} W^{+} W^{-}$. The dependence of the corresponding total cross section on the Higgs boson mass and width is presented in Figure 6 . The shapes of invariant mass $m_{W W}$ 

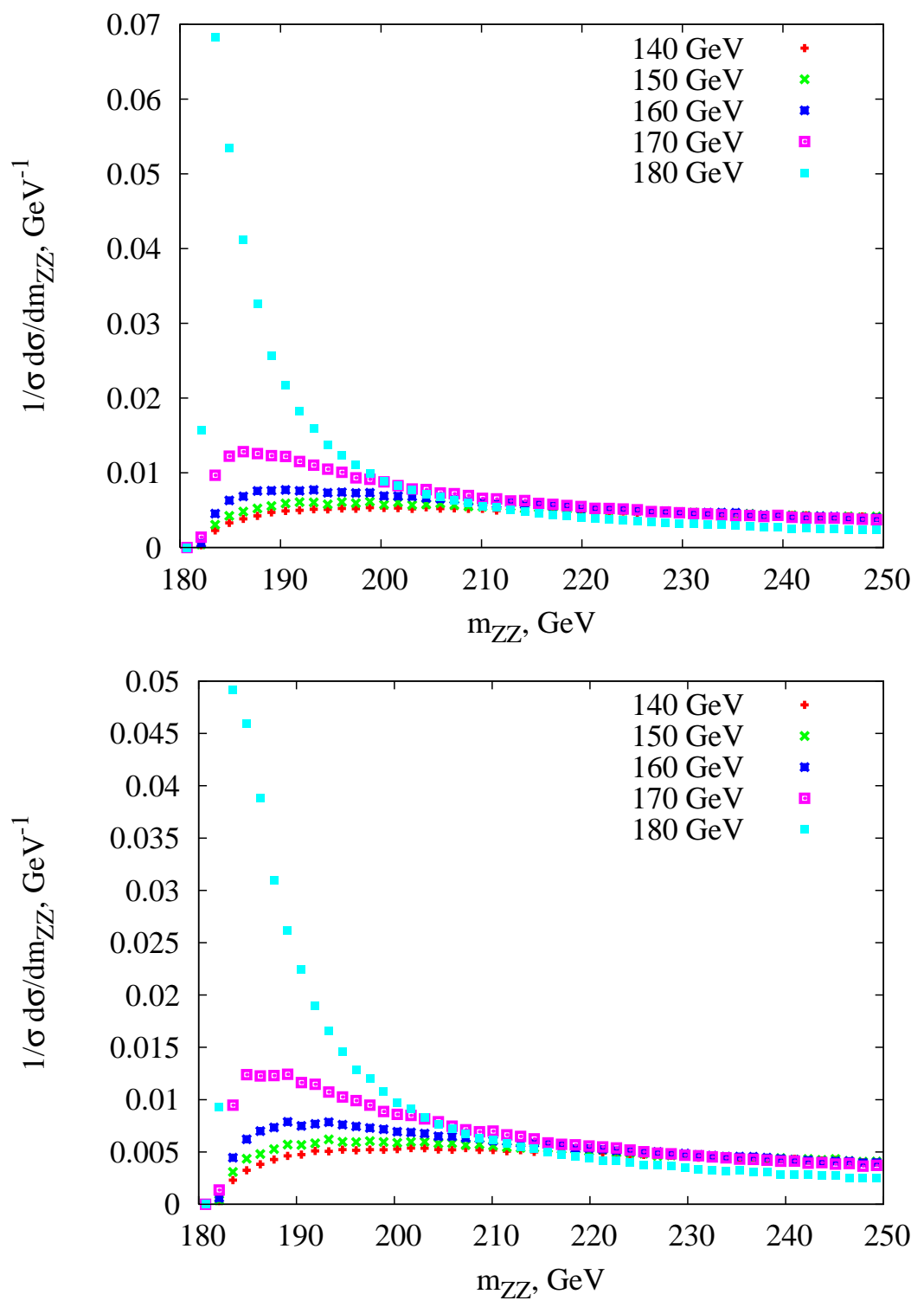

Figure 2: The invariant mass $m_{Z Z}$ distribution for the $p p \rightarrow t \bar{t} Z Z$ at $\sqrt{s}=$ $14 \mathrm{TeV}$ process for several values of the Higgs boson mass for Standard Model Higgs width (upper panel) and for the width which is 8 times larger (lower panel). 

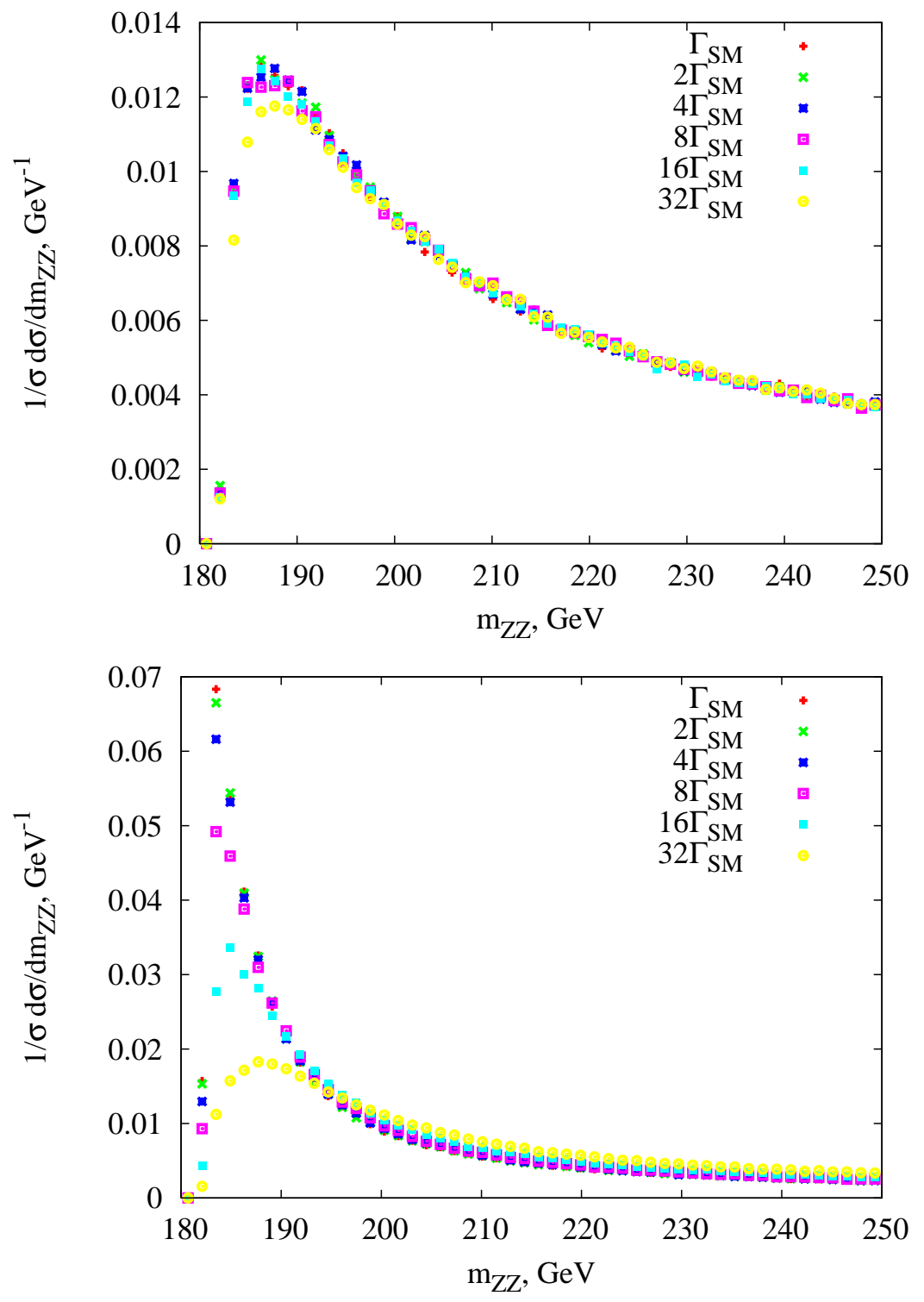

Figure 3: The dependence of the $m_{Z Z}$ invariant mass distribution in $p p \rightarrow$ $t \bar{t} Z Z$ on the width of the Higgs boson for the following values of masses $m_{H}$ : $170 \mathrm{GeV}$ (top panel), $180 \mathrm{GeV}$ (bottom panel), at $\sqrt{s}=14 \mathrm{TeV}$.

distribution are plotted in Figures 7 and 8.

Note in passing, that all our calculations above and below have been 


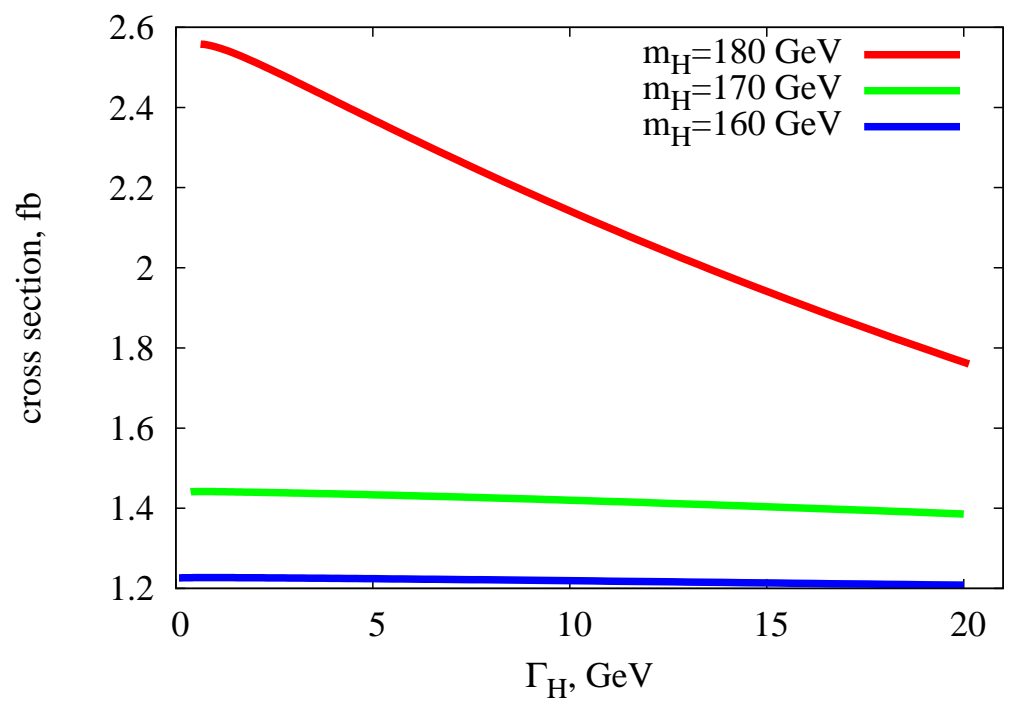

Figure 4: The dependence of the total cross section $p p \rightarrow t \bar{t} Z Z$ at $\sqrt{s}=$ $14 \mathrm{TeV}$ on the width of the Higgs boson for three values of masses $m_{H}$.

done at the leading order in perturbative QCD. We leave a thorough investigation of NLO corrections to future work, which is not straightforward for any $2 \rightarrow 4$ process. In this paper we make a crude estimate of these corrections and study their possible effect on the shape of $m_{Z Z}$ distribution and on the position of its maximum. In all our numerical calculations we take renormalization scale for parton distribution functions to be $Q=M_{Z}$. Here we compare the above results for total cross section and for $m_{Z Z}$ distribution to the results obtained with different choices of $Q$, namely $Q=M_{Z}+M_{t}$ and $Q=\sqrt{\sum_{f} p_{T}^{2}} / 2$, where sum is taken over squared transverse momenta of all particles in the final state. As an example, we consider the case of process $p p \rightarrow t \bar{t} Z Z$ with Standard model Higgs boson $m_{H}=170 \mathrm{GeV}, \sqrt{s}=14 \mathrm{TeV}$. We find for the total cross section of this processes

\begin{tabular}{c|c}
$Q^{2}$ & $\sigma, \mathrm{fb}$ \\
\hline$M_{Z}^{2}$ & 1.44 \\
$\left(M_{Z}+M_{t}\right)^{2}$ & 0.85 \\
$\sum_{f} p_{T}^{2} / 4$ & 1.03
\end{tabular}


The behavior of the cross section with $Q$ is similar to that in $t \bar{t} H$ final state, obtained in Refs. [28, 29], with account of both leading order and next-to-leading order corrections. Thus, one can expect quite a substantial NLO corrections to the total signal cross section. However, as one can see from Figure 5, both the position of maximum and the shape of the $m_{Z Z}$

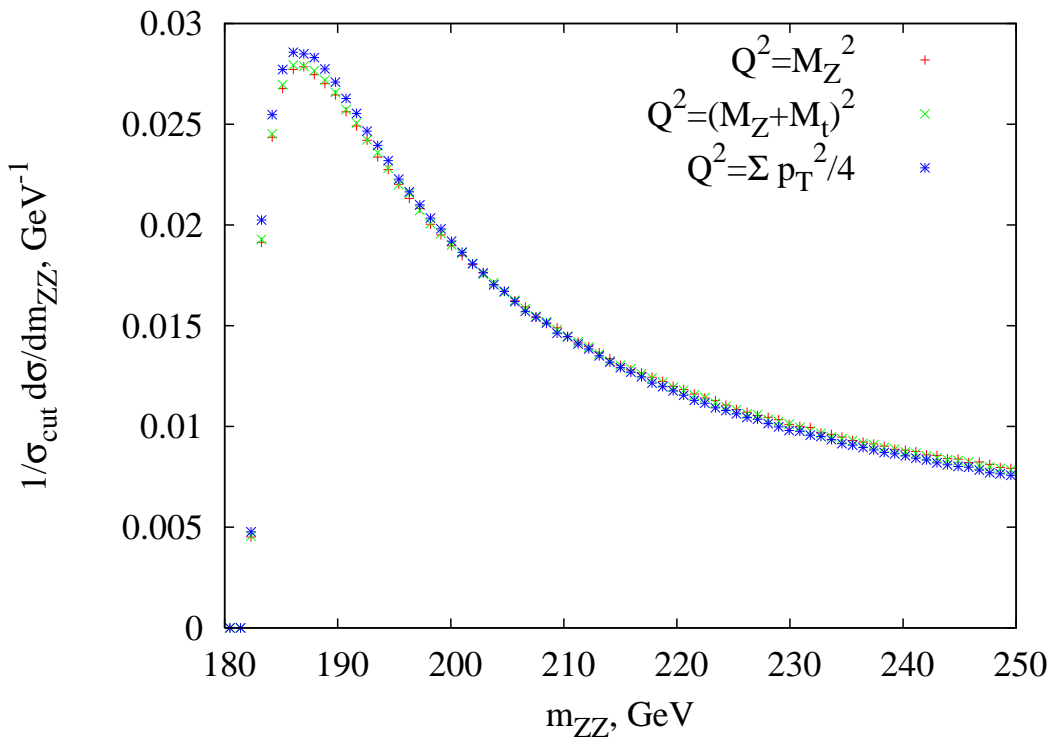

Figure 5: The shapes of the invariant mass $m_{Z Z}$ distribution in $p p \rightarrow t \bar{t} Z Z$ for different choices of renormalization scale at $\sqrt{s}=14 \mathrm{TeV}$.

distribution, normalized to the cross section $\sigma_{\text {cut }}$, obtained by integrating the differential cross section over the relevant interval $180 \mathrm{GeV}<m_{Z Z}<$ $250 \mathrm{GeV}$, remain intact with changing of the renormalization scale in the wide range above. To make a numerical estimate of this uncertainty we evaluate the expected number of events in $7 \mathrm{GeV}$ width bins over $m_{Z Z}$ for the three choices of $Q^{2}$, In a given bin the corresponding three numbers differ by less than 5 percent. This suggests that NLO corrections do not change significantly the shape of this distribution and hence do not add to the uncertainties in the Higgs boson parameters to be determined in the way we propose. 


\section{$3 \quad$ Invisible Higgs in $p p \rightarrow b \bar{b} Z Z$ and $p p \rightarrow b \bar{b} W^{+} W^{-}$ at $\mathrm{LHC}$}

It is worth noting that the total cross sections of the processes with $t$-quarks considered in the previous section are of order of a few fb, which requires high luminosity running of LHC to be of practical interest. The same is true for similar channels $p p \rightarrow b \bar{b} Z Z$ and $p p \rightarrow b \bar{b} W^{+} W^{-}$within the SM. Indeed, in this case the Yukawa coupling of $b$-quarks to Higgs boson is quite small and the diagrams with virtual Higgs boson are suppressed. However, in many promising extensions of the SM this Yukawa coupling increases, as it takes place, for example, in the two-Higgs doublet model (2HD) and in Minimal Supersymmetric Standard Model (MSSM). For illustrative purposes we take the Yukawa coupling of $b$-quarks increased by a factor $A=50$ with respect to the SM case (this refers to the value of $\tan \beta=50$ in 2HD and MSSM). Note, that the change of the $b$-quark Yukawa coupling also yields a change of the Higgs boson total width which we take into account accordingly. In these modifications of the SM with large value of $A$ the Higgs boson width $\Gamma_{m S M}$ is saturated by its decay into $b$-quarks, so that

$$
\Gamma_{m S M}=A^{2} \cdot \Gamma_{S M}^{H \rightarrow b \bar{b}},
$$

where $\Gamma_{S M}^{H \rightarrow b \bar{b}}$ is the SM Higgs boson decay rate into b-quarks.

For the processes with $b$-quarks we exclude from considerations the following regions of the phase space of the final state: $159.3 \mathrm{GeV}<m_{b W^{-}}<$ 189.3 $\mathrm{GeV}$ and $159.3 \mathrm{GeV}<m_{\bar{b} W^{+}}<189.3 \mathrm{GeV}$ because in these regions the cross section is saturated by top-quark production and the interesting effects get obscured. The corresponding total cross sections are given in Figure 9 for $b \bar{b} Z Z$ final state and in Figure 12 for $b \bar{b} W^{+} W^{-}$.

For the processes with $b$-quarks we observe qualitatively similar dependence of the $m_{Z Z}$ distribution on the Higgs boson mass; remarkably, this distribution depends also on the Higgs boson width, see Figures 10 and 11. However, both the shape of $m_{Z Z}$ distribution and the position of its maximum are much more sensitive to $m_{H}$ and $\Gamma_{H}$ as compared to the case of $t$-quarks. So, to obtain the Higgs boson width and mass one should make two-parametric analysis of $m_{Z Z}$ distribution and total cross section. At the same time, with the same collected statistics one can expect to achieve higher accuracy in measurements of the Higgs boson mass and width, than in the channels with $t$-quarks. Of course this is true only for large enough values 
for the constant A.
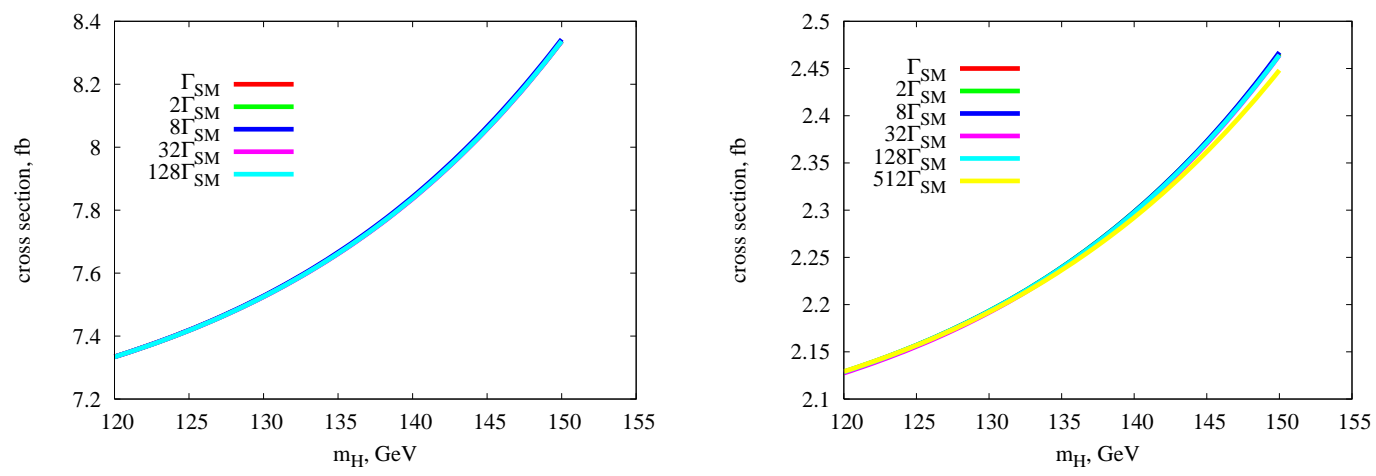

Figure 6: The dependence of the total cross section of $p p \rightarrow t \bar{t} W^{+} W^{-}$at $\sqrt{s}=14 \mathrm{TeV}$ (left) and $\sqrt{s}=10 \mathrm{TeV}$ (right) on the mass of the Higgs boson for various values of the Higgs boson width.

\section{Study of partonic scattering $b \bar{b} \rightarrow Z Z$}

This example demonstrates the main effect under discussion by simple analytical calculation of the three diagrams - $t$ - and $u$-channel $b$-quark exchange and $s$-channel Higgs boson exchange - contributing to $b b \rightarrow Z Z$.

The point is that processes $b \bar{b} \rightarrow Z Z$ and $g g \rightarrow b \bar{b} Z Z$ are closely related. It goes as follows. The b-quark mass is rather small as compared to the discussed range of the invariant mass $M_{Z Z}$. It leads to the well known enhancement of the contribution to the process $g g \rightarrow b \bar{b} Z Z$ from the kinematic region with two very forward b-quarks by the factor $\log \left(M_{Z Z}^{2} / m_{b}^{2}\right)$. This fact allows one to resum the log factors by introducing the b-quark distribution functions in protons and study the process $b \bar{b} \rightarrow Z Z$ with the b-quarks in the initial states (see, $[30,31,32]$ and references therein).

Total partonic cross section of the process $b \bar{b} \rightarrow Z Z$ is obtained with the help of symbolic part of CompHEP and its complete expression is given in Appendix A. Here we present only the zero and the first order terms in $1 / s$ 


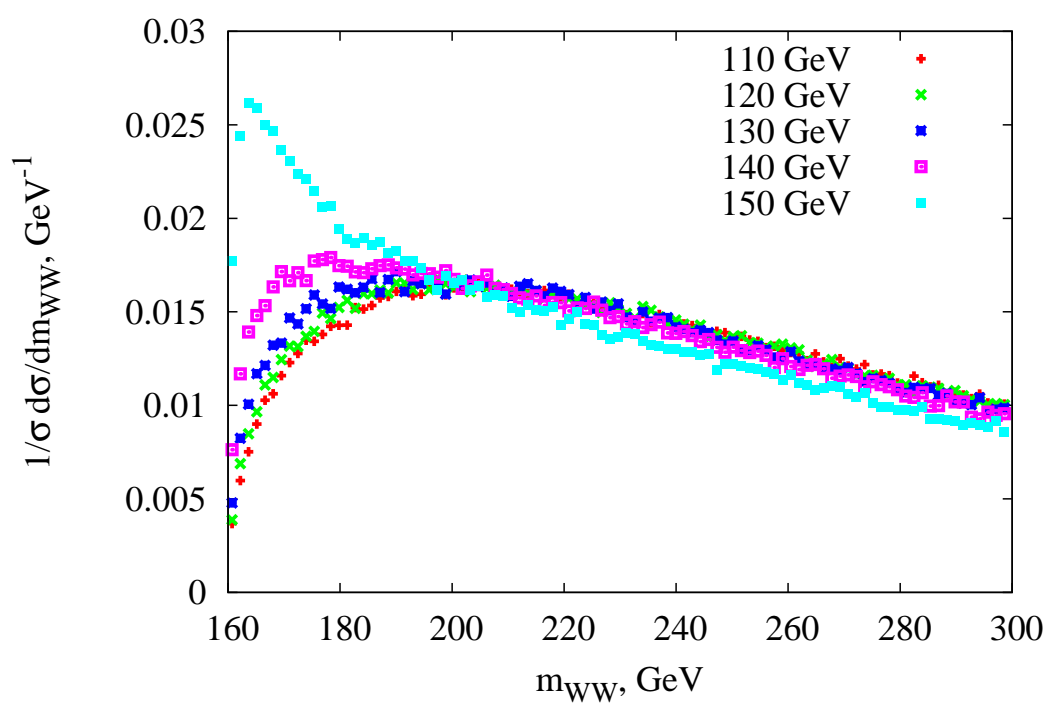

Figure 7: The invariant mass $m_{W W}$ distribution in $p p \rightarrow t \bar{t} W^{+} W^{-}$at $\sqrt{s}=$ $14 \mathrm{TeV}$ for several values of the Higgs boson mass.

(see notations in the Appendix A)

$$
\begin{array}{r}
\sigma_{s}=\frac{\pi \alpha}{6 s_{W}^{4} c_{W}^{4} M_{Z}^{2} s^{2}}\left\{\frac{1}{2}(l-r)^{4} M_{b}^{2} s^{2}+M_{Z}^{4} s\left[2\left(l^{4}+r^{4}\right)\left(\log \frac{s}{M_{b}^{2}}-1\right)\right.\right. \\
\left.-4\left(l^{4}-l^{3} r-l r^{3}+r^{4}\right)\right]+M_{b}^{2} M_{Z}^{2} s\left[2 l r^{2}(l-r)-(l-r)^{4}\right] \\
\left.-(l-r)^{4} M_{b}^{4} s\left(\log \frac{s}{M_{b}^{2}}+1\right)+\frac{1}{4} A(l-r)^{2}\right]-M_{b}^{2} s^{2}+4 M_{b}^{2} M_{Z}^{2} s \\
\left.+M_{b}^{4} s\left(\frac{1}{2} \log \frac{s}{M_{b}^{2}}+2\right)-\left(m_{H}^{2}-\Gamma_{H}^{2}\right) M_{b}^{2} s\right]+\frac{1}{16} A^{2}\left[\frac{1}{2} M_{b}^{2} s^{2}\right. \\
\left.\left.-3 M_{b}^{2} M_{Z}^{2} s-3 M_{b}^{4} s+\frac{1}{2}\left(2 m_{H}^{2}-\Gamma_{H}^{2}\right) M_{b}^{2} s\right]\right\}
\end{array}
$$

The partonic cross section should be converted with parton distribution functions of $b$-quarks in proton $f_{b}\left(x, Q^{2}\right)$ and $f_{\bar{b}}\left(x, Q^{2}\right)$ (where $x$ is the fraction of proton momentum carried by a parton and $Q$ is the characteristic QCD factorization scale taken here equal to be the Higgs mass). This is done with the CompHEP using the b-quark parton distribution functions as given by CTEQ6L1 [25]. 


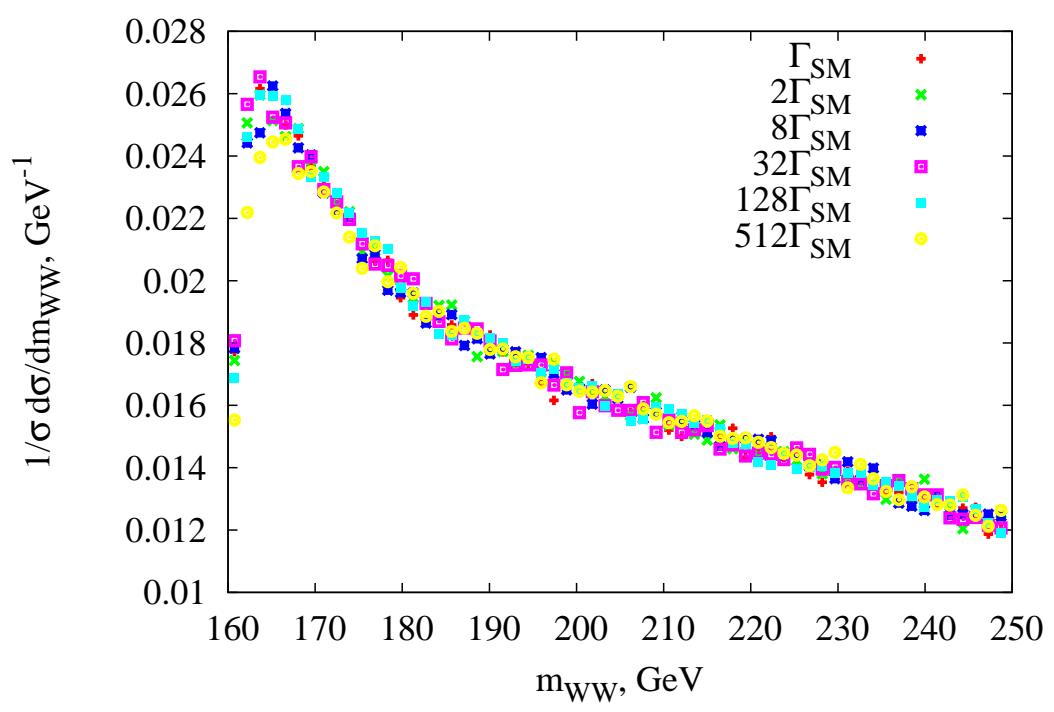

Figure 8: The dependence of the total cross section of $p p \rightarrow t \bar{t} W^{+} W^{-}$at $\sqrt{s}=14 \mathrm{TeV}$ on the width of the Higgs boson for $m_{H}=150 \mathrm{GeV}, \Gamma_{S M}=$ $0.01726 \mathrm{GeV}$.

Then the dependence of the invariant mass $m_{Z Z}$ on mass and width of the Higgs boson for $p p \rightarrow b \bar{b} \rightarrow Z Z$ is presented in Figures 13 and 14. By comparing them with Figs. 10 and 11 we see a nice agreement.

One can understand the behavior of the distribution over $m_{Z Z}$ in the process $p p \rightarrow b \bar{b} Z Z$ from rather general arguments. Let us represent the corresponding matrix element as a sum $M=M_{1}+M_{2}$ where the second term refers to the diagrams describing $Z$-boson pair production via virtual Higgs boson (so, it has the form $M_{2} \sim \frac{1}{m_{Z Z}^{2}-m_{H}^{2}+i m_{Z Z} \Gamma_{H}}$ ), while $M_{1}$ includes all other relevant contributions. Then, integrating partly over the phase space one arrives the integral over invariant mass $m_{Z Z}$,

$$
\begin{array}{r}
\sigma_{s}=\frac{1}{8 \pi} \int d m_{Z Z}^{2} \sqrt{1-\frac{4 m_{Z}^{2}}{m_{Z Z}^{2}}}\left\{f_{11}\left(m_{Z Z}\right)+\frac{2 f_{12}\left(m_{Z Z}\right)\left(m_{Z Z}^{2}-m_{H}^{2}\right)}{\left(m_{Z Z}^{2}-m_{H}^{2}\right)^{2}+\Gamma_{H}^{2} m_{Z Z}^{2}}\right. \\
\left.+\frac{f_{22}\left(m_{Z Z}\right)}{\left(m_{Z Z}^{2}-m_{H}^{2}\right)^{2}+\Gamma_{H}^{2} m_{Z Z}^{2}}\right\} .
\end{array}
$$

Here the square root term comes from two-particle phase space and we omit 

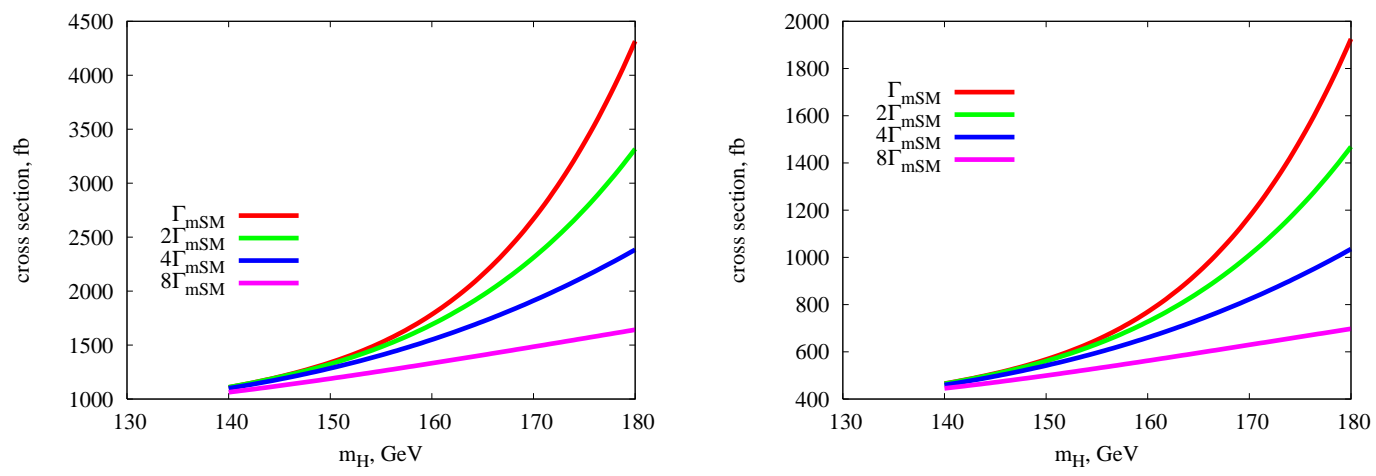

Figure 9: The dependence of the total cross section of $p p \rightarrow b \bar{b} Z Z$ at $\sqrt{s}=$ $14 \mathrm{TeV}$ (left) and $\sqrt{s}=10 \mathrm{TeV}$ (right) on the mass of the Higgs boson in the modified Standard Model with b-Higgs coupling enhanced by factor $A=50$; $\Gamma_{m S M}$ is given by Eq. (1).

the dependence of functions $f_{11}, f_{12}, f_{22}$ on $s$. These functions of $m_{Z Z}$ vary rather mildly in a quite broad interval about $m_{H}$. Treating them as constants one checks that the integrand in Eq. (3) fits the $m_{Z Z}$ invariant mass distribution presented in Section 3. Similar reasoning is valid for other channels under discussion. Thus the behavior of $m_{Z Z}$ distribution observed in this work reflects the Breit-Wigner structure of the virtual Higgs boson contribution.

In the vicinity of the Higgs mass $m_{H}$ the main contribution to the shape of $m_{Z Z}$ invariant mass distribution as a functions of $m_{Z Z}$ comes from the third term in Eq. (3) containing $f_{22}\left(m_{Z Z}\right)$. Hence if properly normalized this distribution approximately does not depend on function $f_{22}\left(m_{Z Z}\right)$ at all (recall we assume that $f_{22}\left(m_{Z Z}\right)$ is a constant in the some interval near $\left.m_{H}\right)$. In this way one can argue that $m_{Z Z}$ invariant mass distribution is not significantly affected by NLO corrections, confirming conclusions of numerical studies in Section 2.

\section{Conclusions}

We have performed a phenomenological analysis of the Higgs boson manifestation at the LHC in the processes of heavy quarks (top and bottom) pair 
production in association with the electroweak bosons $W^{+} W^{-}$and $Z Z$ in the framework of the models where the Higgs boson decay branching rations for SM is practically small (e.g., like in Higgs portal models). In this case one expects that the Higgs boson observation as a resonance is problematic since all the SM decay modes are very much suppressed. However, the Higgs boson should play its role in unitarization of the weak amplitudes behavior, and this property could be exploited to search for the Higgs boson manifestation. We performed complete tree level computations of the relevant $2 \rightarrow 4$ sub-processes numerically and illustrated the main observations by simple symbolic formulas. Numerical analysis is presented for the case of LHC energy of $14 \mathrm{TeV}$ (particular results are given for the energy of $10 \mathrm{TeV}$ for comparison). The total cross sections of the processes $p p \rightarrow t \bar{t} V V$, where $V$ is $Z$ - or $W$-boson, are sensitive to a variation of both the Higgs mass and width in the Higgs mass region somewhat less than the mass of vector boson pair $\left(130-140 \mathrm{GeV}<M_{H}<M_{V V}\right)$. The invariant mass distribution of vector boson pairs also exhibits strong dependence on the Higgs boson mass and width. The processes with the b-quarks $p p \rightarrow b \bar{b} V V$ obviously have too small rate for the case of the SM Higgs coupling, however the processes might be interesting in models where the b-quark coupling to the Higgs boson is significantly increased, for example, in MSSM like models with large $\tan \beta$. In case of $p p \rightarrow b \bar{b} W^{+} W^{-}$one should cut out the phase space region of $\mathrm{W}$ and $\mathrm{b}$ invariant mass around the top quark mass to remove a very large top pair contribution and to observe the effect of cross section and distribution dependence on the Higgs mass and width. We have estimated an impact of possible QCD corrections on observed dependence by performing computations at various QCD renormalization scales. In accordance with known NLO computations for the Higgs production in association with heavy quarks the cross sections and distributions get corrections, however, the observed dependence on the Higgs mass and width is not practically affected. Complete tree level computations of the processes with 4 final state particles allows to take into account all the irreducible backgrounds. However, the influence of reducible backgrounds and effects of detector finite resolutions are needed to be taken into account for more realistic analysis. In particular, if one takes into account subsequent top quark, W- and Z-bosons decays one may study off-shell production and hope to pin down the sensitivity region for the Higgs mass below $130-140 \mathrm{GeV}$. However this looks problematic since rather small rate for considered on-shell production processes will be even smaller in this case. 
Acknowledgments. The work was supported by Russian Ministry of Education and Science under state contract 02.740.11.0244. The work of E.B. was also supported by the grant of Russian Ministry of Education and Science NS-4142.2010.2, RFBR grants 08-02-91002-CERN_a and 08-0292499-CNRSL_a. D.G. thanks the organizers of the long-term workshop in Yukawa Institute YITP-T-10-01 for hospitality. The work of D.G. and S.D. was supported in part by the Russian Foundation for Basic Research (grants 08-02-00473a), by the grants of the President of the Russian Federation NS5525.2010.2 and by FAE program (government contract П520). The work of S.D. was also supported by the grants of the President of the Russian Federation MK-4317.2009.2, by FAE program (government contract П2598). Numerical part of the work was performed on the Computational cluster of the Theoretical Division of INR RAS.

\section{A Formula for cross section $b \bar{b} \rightarrow Z Z$}

Let us introduce following notations

$$
\begin{array}{r}
s_{W}=\sin \theta_{W}, \quad l=T_{3}-Q s_{W}^{2} \equiv-\frac{1}{2}+\frac{1}{3} s_{W}^{2}, \quad r=-Q s_{W}^{2} \equiv \frac{1}{3} s_{W}^{2}, \\
A_{s}(s)=\sqrt{s-4 M_{b}^{2}} \sqrt{s-4 M_{Z}^{2}}, \quad U(s)=s+A_{s}(s)-2 M_{Z}^{2}, \\
D(s)=s-A_{s}(s)-2 M_{Z}^{2} .
\end{array}
$$




$$
\begin{gathered}
\sigma_{s}=\frac{\pi \alpha}{6 s_{W}^{4} c_{W}^{4} M_{Z}^{2} s^{2}}\left\{( \frac { 1 } { D } - \frac { 1 } { U } ) \left[-8\left(l^{4}+r^{4}\right) M_{Z}^{8}+8 M_{Z}^{6} M_{b}^{2}\left(l^{4}-5 l^{3} r+2 l^{2} r^{2}-5 l r^{3}\right.\right.\right. \\
\left.\left.+r^{4}\right)-2 M_{Z}^{4} M_{b}^{4}\left(l^{4}-20 l^{3} r+54 l^{2} r^{2}-20 l r^{3}+r^{4}\right)-8 M_{b}^{2} M_{Z}^{4} s\left(l^{4}-l^{3} r-l r^{3}+r^{4}\right)\right] \\
+\frac{1}{2 M_{Z}^{2}-s} \log \frac{U}{D}\left[-8\left(l^{4}+r^{4}\right) M_{Z}^{8}+4 M_{Z}^{6} M_{b}^{2}\left(6 l^{4}-10 l^{3} r+17 l^{2} r^{2}-11 l r^{3}+6 r^{4}\right)\right. \\
-2 M_{Z}^{4} M_{b}^{4}\left(3 l^{4}-12 l^{3} r+2 l^{2} r^{2}-12 l r^{3}+3 r^{4}\right)-8 M_{b}^{8}\left(l^{4}+r^{4}\right)+4 M_{Z}^{4} M_{b}^{2} s\left(l^{4}+2 l^{3} r\right. \\
\left.-11 l^{2} r^{2}+3 l r^{3}+r^{4}\right)+\frac{3}{2} M_{b}^{4} M_{Z}^{2} s\left(l^{4}-8 l^{3} r+14 l^{2} r^{2}-8 l r^{3}+r^{4}\right) \\
+A_{s}(s)\left[-2\left(l^{4}+r^{4}\right) M_{Z}^{4}+M_{Z}^{4}+r_{b}^{2}\left(2 l^{2} r^{2}-2 l r^{3}\right)+\frac{1}{2}(l-r)^{4} M_{b}^{2} s\right] \\
+\frac{A M_{b}^{2}\left(s-m_{H}^{2}\right)}{\left(s-m_{H}^{2}\right)^{2}+s \Gamma_{H}^{2}}\left[A_{s}(s)\left(-\frac{1}{4}(l-r)^{2} s^{2}+\frac{1}{2}(l-r)^{2} M_{Z}^{2} s-2\left(l^{2}+r^{2}\right) M_{Z}^{4}\right)\right. \\
+\log \frac{U}{D}\left(-4(l-r)^{2} M_{Z}^{4} M_{b}^{2}+\frac{1}{2}(l-r)^{2} M_{b}^{2} s^{2}-2(l-r)^{2} M_{b}^{2} M_{Z}^{2} s-2\left(l^{2}+r^{2}\right) M_{Z}^{6}\right. \\
\left.\left.\left.+4\left(l^{2}-l r+r^{2}\right) M_{Z}^{4} s\right)\right]+\frac{A^{2} M_{b}^{2} A_{s}(s)}{32\left(\left(s-m_{H}^{2}\right)^{2}+s \Gamma_{H}^{2}\right)}\left(s-4 M_{b}^{2}\right)\left(12 M_{Z}^{4}-4 M_{Z}^{2} s+s^{2}\right)\right\}
\end{gathered}
$$

Here $s=\left(p_{1}+p_{2}\right)^{2}$ where $p_{1}$ and $p_{2}$ are the momenta of $b$ and $\bar{b}$ quarks, respectively. One can check that the leading terms in $s$ (which lead to constant cross section at high energies, thus violating unitarity) cancel at $A=1$, otherwise they should be canceled by terms coming from other non-SM particles.

We recall that the width of the Higgs boson $\Gamma_{H}$ depends on the amplification factor $A$ as well.

\section{References}

[1] C. Amsler et al. [Particle Data Group], Phys. Lett. B 667, 1 (2008).

[2] J. McDonald, Phys. Rev. D 50, 3637 (1994) [arXiv:hep-ph/0702143]; C. P. Burgess, M. Pospelov and T. ter Veldhuis, Nucl. Phys. B 619, 709 (2001) [arXiv:hep-ph/0011335]. 
[3] B. Patt and F. Wilczek, "Higgs-field portal into hidden sectors," arXiv:hep-ph/0605188.

[4] R. E. Shrock and M. Suzuki, Phys. Lett. B 110 (1982) 250.

[5] N. Arkani-Hamed, S. Dimopoulos and G. R. Dvali, Phys. Lett. B 429, 263 (1998) [arXiv:hep-ph/9803315].

[6] L. Randall and R. Sundrum, Phys. Rev. Lett. 83 (1999) 3370 [arXiv:hepph/9905221].

[7] K. Belotsky, D. Fargion, M. Khlopov, R. Konoplich and K. Shibaev, Phys. Rev. D 68 (2003) 054027 [arXiv:hep-ph/0210153].

[8] N. V. Krasnikov, Mod. Phys. Lett. A 13 (1998) 893 [arXiv:hep$\mathrm{ph} / 9709467]$.

[9] Y. Hosotani, K. Oda, T. Ohnuma and Y. Sakamura, Phys. Rev. D 78 (2008) 096002 [Erratum-ibid. D 79 (2009) 079902] [arXiv:0806.0480 [hep-ph]].

[10] L. Fromme, S. J. Huber and M. Seniuch, JHEP 0611, 038 (2006) [arXiv:hep-ph/0605242].

[11] T. Binoth and J. J. van der Bij, Z. Phys. C 75, 17 (1997) [arXiv:hep$\mathrm{ph} / 9608245]$.

[12] S. Bock, R. Lafaye, T. Plehn, M. Rauch, D. Zerwas and P. M. Zerwas, "Measuring Hidden Higgs and Strongly-Interacting Higgs Scenarios," arXiv:1007.2645 [hep-ph].

[13] O. J. P. Eboli and D. Zeppenfeld, Phys. Lett. B 495 (2000) 147 [arXiv:hep-ph/0009158].

[14] J. F. Gunion, Phys. Rev. Lett. 72 (1994) 199 [arXiv:hep-ph/9309216].

[15] D. Choudhury and D. P. Roy, Phys. Lett. B 322 (1994) 368 [arXiv:hep$\mathrm{ph} / 9312347]$.

[16] R. M. Godbole, M. Guchait, K. Mazumdar, S. Moretti and D. P. Roy, Phys. Lett. B 571 (2003) 184 [arXiv:hep-ph/0304137]. 
[17] B. W. Lee, C. Quigg and H. B. Thacker, Phys. Rev. D 16, 1519 (1977).

[18] T. Appelquist and M. S. Chanowitz, Phys. Rev. Lett. 59, 2405 (1987) [Erratum-ibid. 60, 1589 (1988)].

[19] M. S. Chanowitz, M. A. Furman and I. Hinchliffe, Nucl. Phys. B 153, 402 (1979).

[20] J. Bagger et al., Phys. Rev. D 52, 3878 (1995) [arXiv:hep-ph/9504426].

[21] H. Davoudiasl, T. Han and H. E. Logan, Phys. Rev. D 71, 115007 (2005) [arXiv:hep-ph/0412269].

[22] A. Pukhov et al., "CompHEP: A package for evaluation of Feynman diagrams and integration over multi-particle phase space. User's manual for version 33," arXiv:hep-ph/9908288.

[23] E. Boos et al. [CompHEP Collaboration], Nucl. Instrum. Meth. A 534 (2004) 250 [arXiv:hep-ph/0403113].

[24] http://comphep.sinp.msu.ru/

[25] J. Pumplin, D. R. Stump, J. Huston, H. L. Lai, P. M. Nadolsky and W. K. Tung, JHEP 0207 (2002) 012 [arXiv:hep-ph/0201195].

[26] A. Djouadi, J. Kalinowski and M. Spira, Comput. Phys. Commun. 108 (1998) 56 [arXiv:hep-ph/9704448].

[27] J. Alcaraz [ALEPH Collaboration and CDF Collaboration and D0 Collaboration and an], "Precision Electroweak Measurements and Constraints on the Standard Model," arXiv:0911.2604 [hep-ex].

[28] W. Beenakker, S. Dittmaier, M. Kramer, B. Plumper, M. Spira and P. M. Zerwas, Nucl. Phys. B 653, 151 (2003) [arXiv:hep-ph/0211352].

[29] S. Dawson, C. Jackson, L. H. Orr, L. Reina and D. Wackeroth, Phys. Rev. D 68, 034022 (2003) [arXiv:hep-ph/0305087].

[30] F. Maltoni, Z. Sullivan and S. Willenbrock, Phys. Rev. D 67, 093005 (2003) [arXiv:hep-ph/0301033].

[31] E. Boos and T. Plehn, Phys. Rev. D 69, 094005 (2004) [arXiv:hep$\mathrm{ph} / 0304034]$. 
[32] J. M. Campbell et al., "Higgs boson production in association with bottom quarks," arXiv:hep-ph/0405302. 

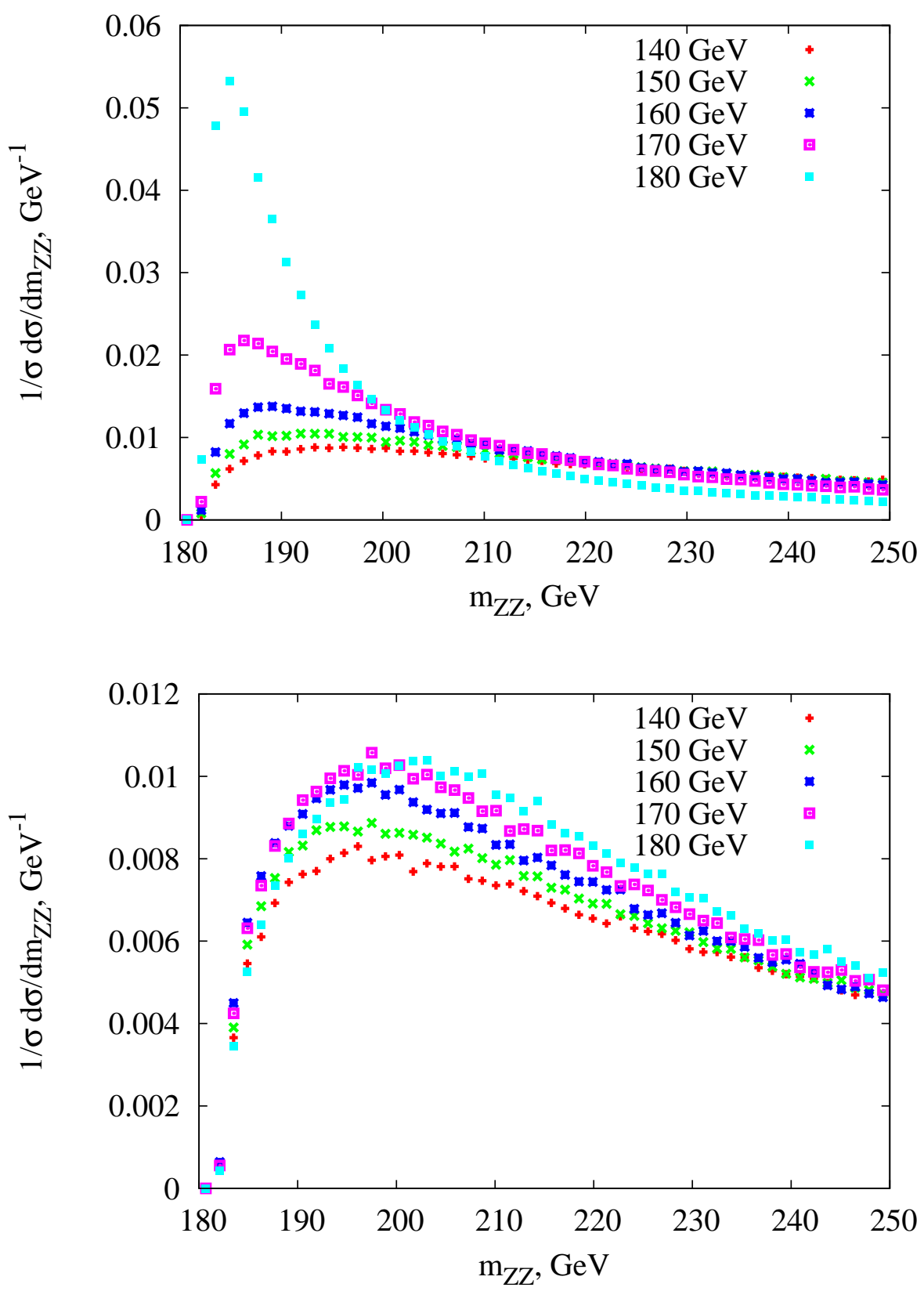

Figure 10: The invariant mass $m_{Z Z}$ distribution in the $p p \rightarrow b \bar{b} Z Z$ process for several values of the Higgs boson mass for modified Standard Model Higgs boson width $\Gamma_{m S M}$ given by Eq. (1) (upper panel) and for the width 8 times larger due to invisible decay mode (lower panel). 

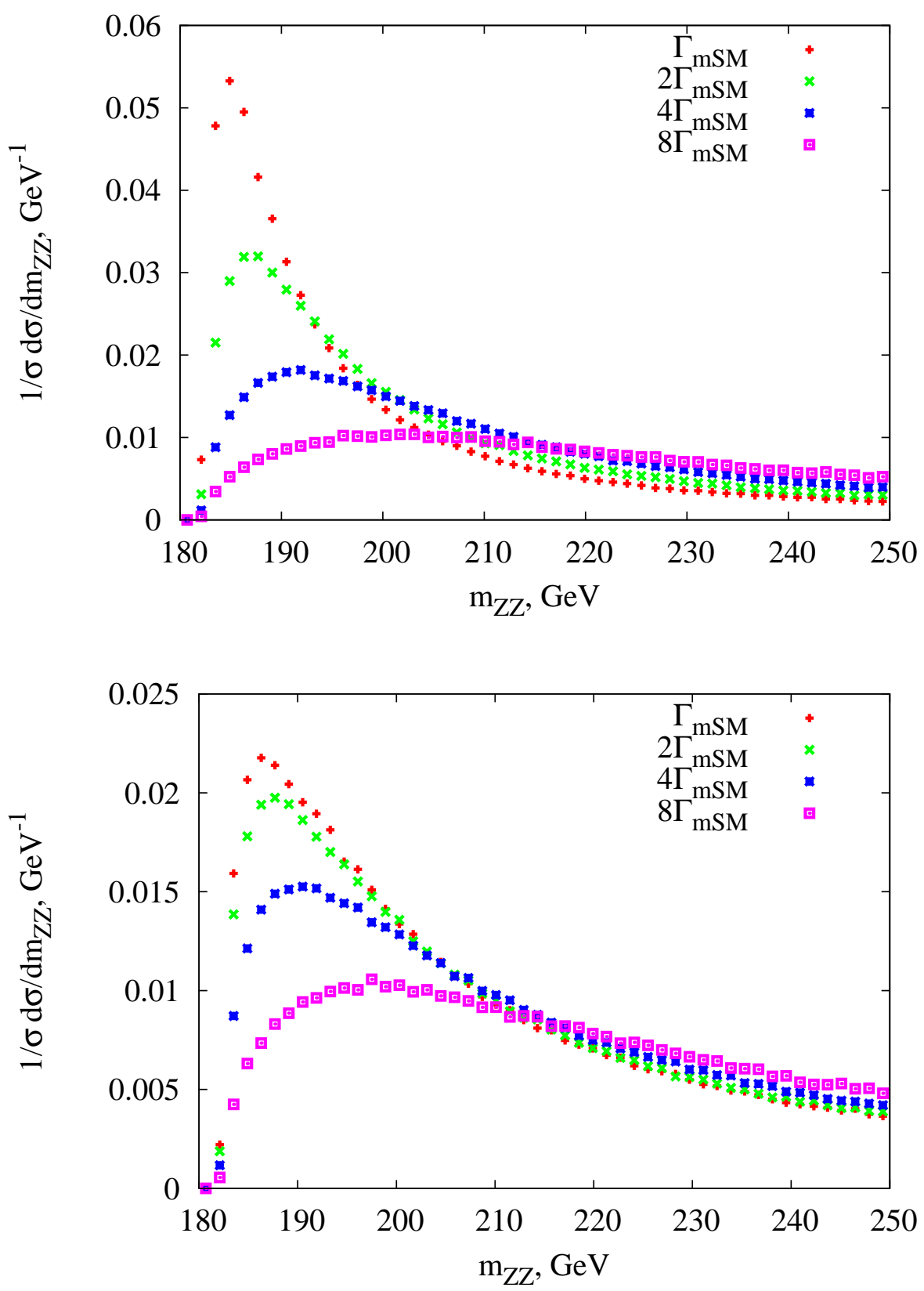

Figure 11: The dependence of the total cross section of $p p \rightarrow b \bar{b} Z Z$ on the width of the Higgs boson for $m_{H}=180 \mathrm{GeV}, \Gamma_{m S M}=9.04 \mathrm{GeV}$ (upper panel) and $m_{H}=170 \mathrm{GeV}, \Gamma_{m S M}=8.41 \mathrm{GeV}$ (lower panel). $\Gamma_{m S M}$ is given by Eq. (1) with factor $A=50$. 

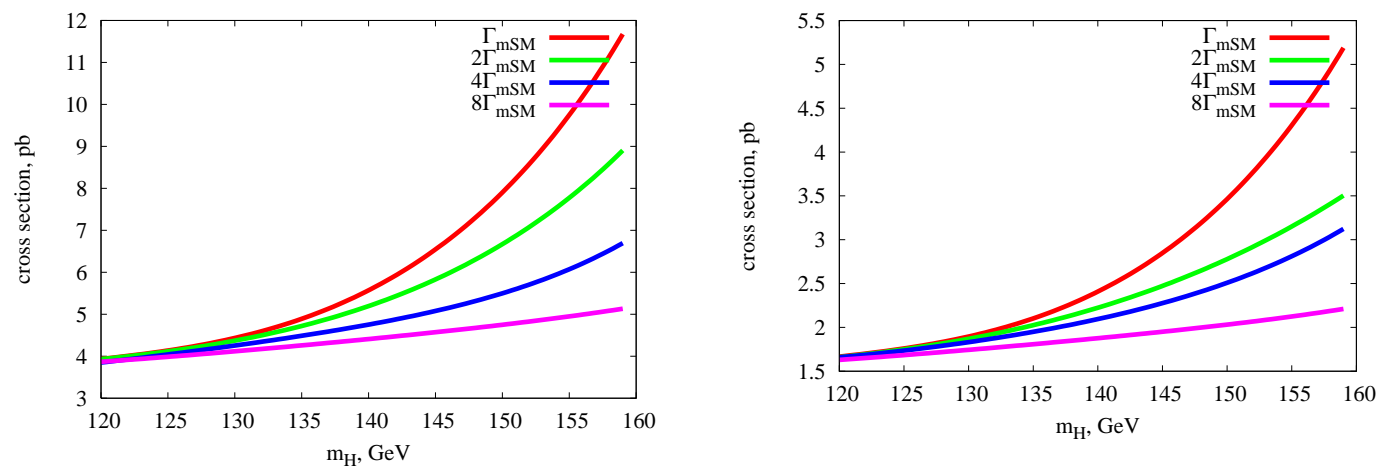

Figure 12: The dependence of the total cross section of $p p \rightarrow b \bar{b} W^{+} W^{-}$at $\sqrt{s}=14 \mathrm{TeV}$ (left panel) and $\sqrt{s}=10 \mathrm{TeV}$ (right panel) on the mass of the Higgs boson in the modified Standard Model with b-Higgs coupling enhanced by factor $A=50 ; \Gamma_{m S M}$ is given by Eq. (1). We exclude the following part of the phase space: $159.3 \mathrm{GeV}<M_{b W^{+}}<189.3 \mathrm{GeV}, 159.3 \mathrm{GeV}<M_{\bar{b} W^{-}}<$ $189.3 \mathrm{GeV}$. 

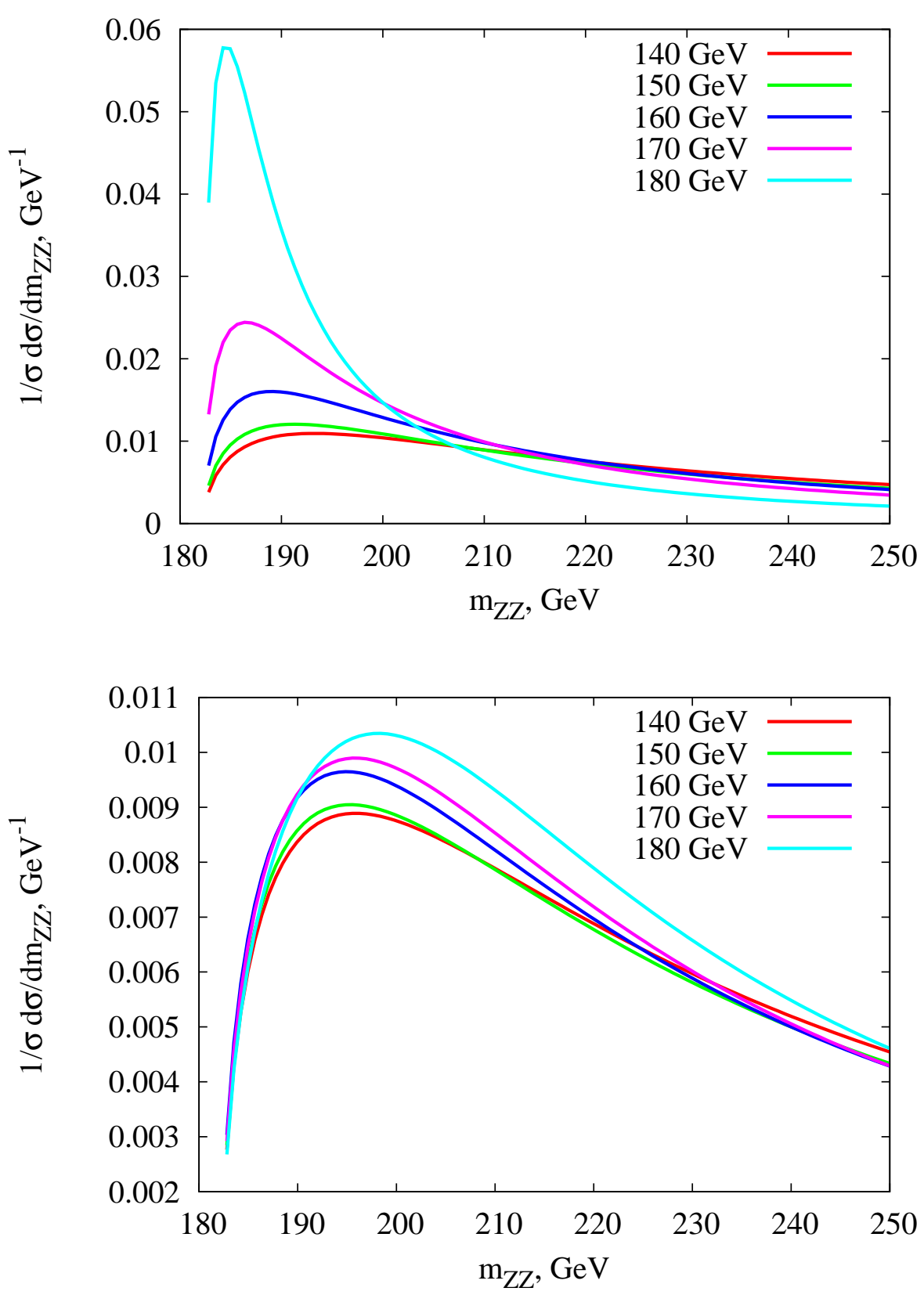

Figure 13: The invariant mass $m_{Z Z}$ distribution for the $b \bar{b} \rightarrow Z Z$ process for several values of the Higgs boson mass in the modified SM with enhanced b-Higgs coupling by factor $A=50$, see Eq. (1), for the Higgs boson width $\Gamma_{m S M}$ (upper panel) and for the width 8 times larger (lower panel) calculated with analytical formula (6). 

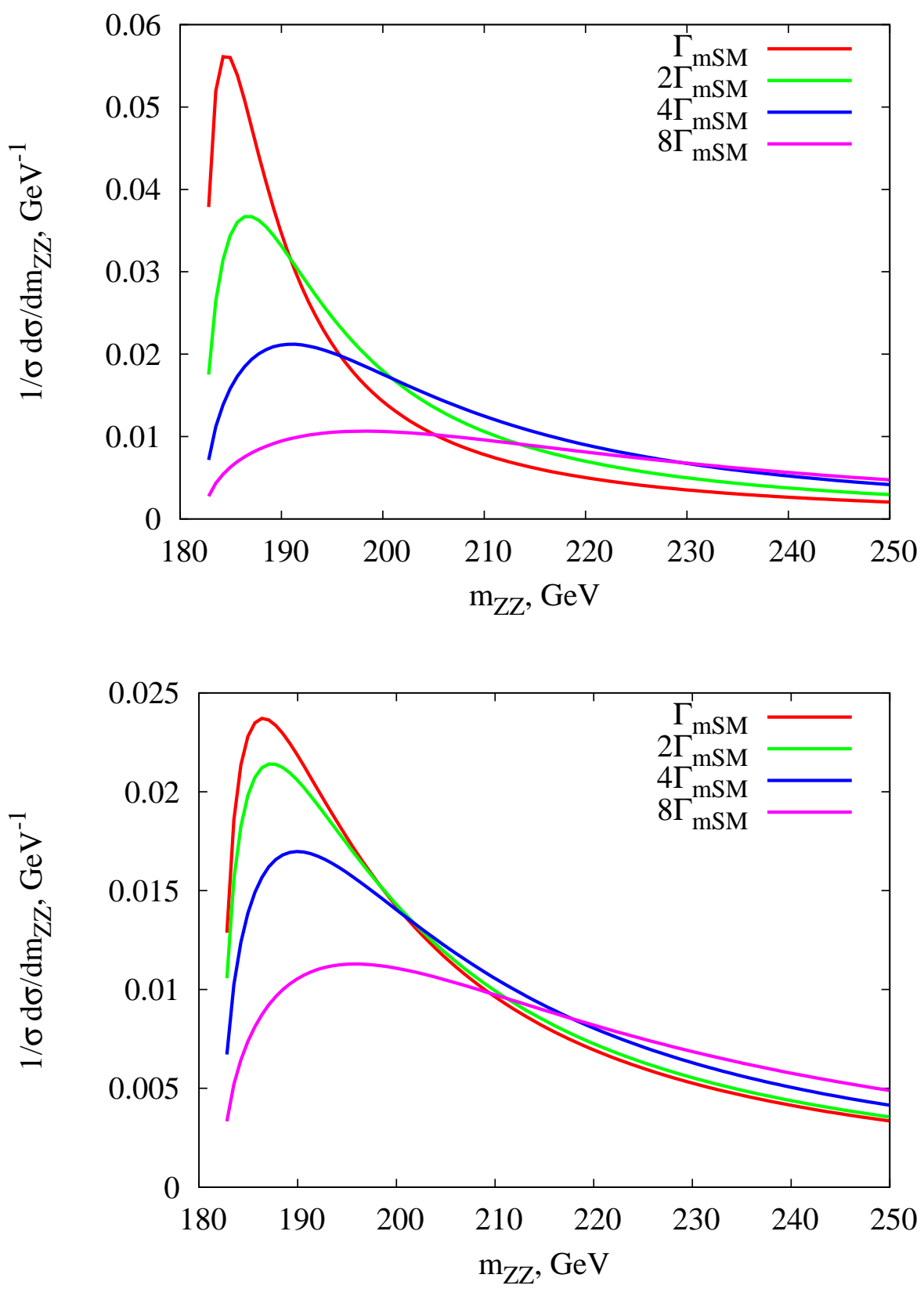

Figure 14: The dependence of the total cross section $b \bar{b} \rightarrow Z Z$ on the width of the Higgs boson in the modified SM with enhanced b-Higgs coupling by factor $A=50$, see Eq. (1), for $m_{H}=180 \mathrm{GeV}, \Gamma_{H}=9.04 \mathrm{GeV}$ (upper panel) and $m_{H}=170 \mathrm{GeV}, \Gamma_{H}=8.41 \mathrm{GeV}$ (lower panel) calculated with analytical formula (6). 